\title{
Fluxon density waves in long Josephson junctions
}

\author{
Olsen, O. H.; Ustinov, A. V.; Pedersen, Niels Falsig
}

Published in:

Physical Review B

Link to article, DOI:

10.1103/PhysRevB.48.13133

Publication date:

1993

Document Version

Publisher's PDF, also known as Version of record

Link back to DTU Orbit

Citation (APA):

Olsen, O. H., Ustinov, A. V., \& Pedersen, N. F. (1993). Fluxon density waves in long Josephson junctions. Physical Review B, 48(17), 13133-13136. https://doi.org/10.1103/PhysRevB.48.13133

\section{General rights}

Copyright and moral rights for the publications made accessible in the public portal are retained by the authors and/or other copyright owners and it is a condition of accessing publications that users recognise and abide by the legal requirements associated with these rights.

- Users may download and print one copy of any publication from the public portal for the purpose of private study or research.

- You may not further distribute the material or use it for any profit-making activity or commercial gain

- You may freely distribute the URL identifying the publication in the public portal

If you believe that this document breaches copyright please contact us providing details, and we will remove access to the work immediately and investigate your claim. 


\title{
Fluxon density waves in long Josephson junctions
}

\author{
O. H. Olsen \\ Biostructure Department, Novo-Nordisk Research Institute, DK-2880 Bagsvaerd, Denmark \\ A. V. Ustinov* and N. F. Pedersen \\ Physics Laboratory I, Technical University of Denmark, DK-2800 Lyngby, Denmark
}

(Received 23 June 1993)

\begin{abstract}
Numerical simulations of the multiple fluxon dynamics stimulated by an external oscillating force applied at a boundary of a long Josephson junction are presented. The calculated $I-V$ characteristics agree well with a recent experimental observation of rf-induced satellite flux-flow steps. The voltage spacing between the satellites and the main flux-flow step corresponds to the second harmonic of the external frequency. We find that the satellite flux-flow modes are characterized by collective excitations propagating through the fluxon chain. These dynamical states can be interpreted as fluxon density waves.
\end{abstract}

Dynamics of fluxons (magnetic flux quanta) in quasione-dimensional long Josephson tunnel junctions (of the length $L \gg \lambda_{J}$, where $\lambda_{J}$ is the Josephson penetration depth) has received increasing interest over the last decade as a model system for soliton physics and because of possible applications in superconducting electronics. ${ }^{1}$ In this paper we focus on multiple fluxon properties and study new phenomena which involve collective motion of a large number of fluxons.

New resonant regimes were found in recent millimeterwave experiments ${ }^{2}$ with a long junction biased in the flux-flow mode. In $I-V$ characteristics, besides an ordinary flux-flow step (FFS), satellite flux-flow steps appear under the influence of external $\mathrm{rf}$ radiation. The voltage spacing between the satellites corresponds to twice that of the Shapiro step spacing for the external rf frequency $f_{\text {ext }}$. In contrast to the ordinary rf-induced Shapiro steps, the voltage position of the satellite steps was found to be monotonically changing with applied magnetic field.

A formal explanation for flux-flow satellite steps would be the mixing between the flux-flow frequency and the external frequency $f_{\text {ext }}$. A similar effect was found previously for Fiske steps in small Josephson junctions. ${ }^{3}$ However, one has to understand why in the case of the long Josephson junction only the second harmonic of $f_{\text {ext }}$ appears between the mixing products. A qualitative explanation of high satellite steps (HSS's) as due to rfassisted reflections of fluxons into antifluxons at the junction boundary has been tentatively suggested in another paper. ${ }^{2}$ However, no explanation based on the fluxonantifluxon model has been found for low satellite steps (LSS's). Flux-flow satellite regimes have not been attempted to be investigated numerically yet. The aim of the present work is to study numerically the physical mechanism responsible for these regimes.

A long Josephson junction is described by the perturbed sine-Gordon equation

$$
\varphi_{x x}-\varphi_{t t}=\sin \varphi+\alpha \varphi_{t}-\gamma,
$$

where $\varphi(x, t)$ is the superconducting phase difference between the electrodes of the junction, the spatial coordi- nate $x$ is normalized to $\lambda_{J}$, the time $t$ is normalized to the inverse plasma frequency $\omega_{0}^{-1}, \alpha$ is the dissipation coefficient, and $\gamma$ is the normalized bias current density. We investigate the particular case when the system described by Eq. (1) is placed in the following boundary conditions:

$$
\varphi_{x}(0, t)=h, \quad \varphi_{x}(\ell, t)=h+h_{\mathrm{ext}}(t),
$$

where $h_{\text {ext }}(t)=a_{\text {ext }} \sin \omega_{\text {ext }} t, \ell=L / \lambda_{J}$ is the normalized length of the Josephson junction, $h$ is the normalized external dc magnetic field, $a_{\text {ext }}$ is the normalized amplitude of the external ac field, and $\omega_{\text {ext }}$ is its angular frequency normalized to $\omega_{0}$. The boundary conditions (2) correspond to the experimental situation ${ }^{2}$ where the junction was placed symmetrically in the external dc and asymmetrically in the external ac magnetic fields.

The numerical procedure applied to solve the system described by Eq. (1) with the boundary conditions given by Eqs. (2) is based on a "leap-frog" scheme. In the $I-V$ characteristics shown in the following the averaged voltage $\tilde{V}=\langle d \varphi / d t\rangle$ has been normalized to the voltage of the first zero field step $\tilde{V}_{\mathrm{ZFS} 1}=2 \pi / \ell$, thus $V=\tilde{V} / \tilde{V}_{\mathrm{ZFS} 1}$. After calculation of the normalized voltage $V$ for a particular value of $\gamma$ the computations are stopped and then restarted with the initial values now being the previous solution and $\gamma$ being gradually increased. This procedure is continued for $\gamma \leq 1.0$ with $\Delta \gamma=0.002$. When $\gamma=1.0$ is reached the sign of $\Delta \gamma$ is changed and the procedure is repeated until $\gamma=0$ is obtained. For the simulations we have used the values of parameters close to that in the experiment. ${ }^{2}$ The normalized values were the following: $\ell=15, \alpha=0.08, h=3-6, a_{\text {ext }}=0-3, \omega_{\text {ext }}=1.4$. In order to save simulation time the value for the dissipation parameter $\alpha$ was chosen to be by a factor of about 3-4 larger than in the experiment. We also performed a series of runs with lower $\alpha$ and found no qualitative difference from the results presented below.

A multiple fluxon state in a long Josephson junction is realized in a sufficiently large dc magnetic field $h>2$. In this case fluxons penetrate into the junction and the normalized spacing between them becomes $\xi \approx 2 \pi / h$. The 
fluxon motion with the velocity $v$ close to the Swihart velocity $\bar{c}$ can be detected experimentally by observing the FFS in the $I-V$ characteristics. ${ }^{4}$ The step appears at the normalized voltage

$$
V=V_{f f} \approx \frac{\ell}{\xi} .
$$

The calculated $I-V$ characteristics of the junction with $h=6$ and $a_{\text {ext }}=0$ displayed a flux-flow step at $V \approx 14$. This voltage is in fairly good agreement with Eq. (3) which yields $V_{f f}=14.32$. The $I-V$ characteristics corresponding to the same value of $h=6$ but with the external oscillating force amplitude $a_{\text {ext }}=2.5$ are shown in Fig. 1(a). The FFS at $V_{f f} \approx 14$ still remains; however, its current amplitude is suppressed. In contrast to the case with $a_{\text {ext }}=0$, two satellite groups of steps appear on both sides from the FFS. The LSS appears at $V_{f f-1} \approx 7.5$ and the HSS is seen at $V_{f f+1} \approx 21$. As the original FFS, the satellites are also split into several (two or three) closely spaced steps due to Fiske resonances which appear because the damping in the junction is not sufficiently large and the reflected wave attenuation criterion $\alpha \ell \gg 1$ is not satisfied. ${ }^{4}$ The reflected wave is excited when the fluxons reach the boundary $x=\ell$. Figure 1(c) shows the $I-V$ characteristics calculated for the same ac amplitude $a_{\text {ext }}=2.5$ but for a slightly lower dc magnetic field $h=5$. This figure shows typical behavior of satellite steps for varying the dc magnetic field $h$. The FFS moves from $V \approx 14$ to $V \approx 11.5$ in agreement with Eq. (3). In a similar manner, the voltage positions of the satellite steps change with magnetic field. However, the voltage spacing between the satellites and the FFS, $\Delta V_{\text {sat }} \approx 6.7$, remains unchanged.

The most well-known phenomenon which characterizes a Josephson junction placed under external electromagnetic radiation is the appearance of $\mathrm{rf}$-induced or, so-called, Shapiro steps in the $I-V$ characteristics. ${ }^{5}$ According to the Josephson relation, the normalized voltage spacing between the conventional Shapiro steps is $\Phi_{0} \omega_{\text {ext }} / 2 \pi$, where $\Phi_{0}$ is the magnetic flux quantum and $f_{\text {ext }}$ is the frequency of the radiation. In normalized units for the external force applied with $\omega_{\text {ext }}=1.4$ the Shapiro step voltages are expected to be $V_{n}=n V_{\omega}=$ $n \omega_{\text {ext }} \ell / 2 \pi \approx 3.34 n$. Indeed, in the simulated curves shown in Fig. 1 one can see a small vertical step shown by an arrow. This step corresponds to the first Shapiro step at $V=V_{\omega}$. A detailed analysis of the phase dynamics in this step indicates that it corresponds to the phase-locked state of the junction to the external ac drive. As expected, the Shapiro step voltage position does not change with magnetic field, i.e., from curve (a) to (b).

In agreement with experiment ${ }^{2}$ the voltage spacing between the calculated FFS and the satellites gives approximately $2 V_{\omega}$. We have calculated $I-V$ curves for different external frequencies $0.9<\omega_{\text {ext }}<2$ and found similar results. For the larger amplitudes $a_{\text {ext }}$ we have seen higher satellites at $V=V_{f f+2}$ and $V=V_{f f+3}$ which have been also detected in the experiment. In Figure 1(a) the second HSS is noticeable as a small step at $V=V_{f f+2} \approx 28$. Fig. 1(b) shows that this step moves with magnetic field as expected.

In order to understand the fluxon dynamics in the junction associated with the flux-flow satellites we have investigated the spatially temporal phase pattern in the junction. The conventional way of doing that is to plot the evolution of the current $\varphi_{x}(x, t)$ or the voltage $\varphi_{t}(x, t)$. However, this method was found not to be sufficient to understand the complicated dynamic states in the junction. Because of the large amplitude oscillations in the junction induced by the external drive with $\omega_{\text {ext }}>1$, it was difficult to distinguish between these modes and the fluxons moving in the junction. Instead, looking at the individual fluxon trajectories was found to be the most informative. In this case we plotted the spatial and time dependence of the points in the junction where $\varphi(x, t)=\varphi_{m}=2 \pi m+\pi$, with $m$ being an arbitrary integer. These points approximately correspond to the locations of fluxon centers of mass in the junction.

For the FFS [point $A$ in Fig. 1(a)] the pattern $\varphi_{m}(x, t)$ is shown in Fig. 2(a). Each line corresponds to a fluxon trajectory. The slope of the trajectories gives the fluxon velocity $v$ which is very close to 1 at the FFS. In order to justify the correlation between the external force $h_{\text {ext }}(t)$ and the phase dynamics of the junction, $h_{\text {ext }}(t)$ is shown to the right from each plot with the same time scale. The dynamic states of the LSS and the HSS corresponding to points $B$ and $C$ in Fig. 1(a) are shown in Figs. 2(b),(c). For these regimes the modulation of the fluxon trajectories is seen to be very strong. The average number of
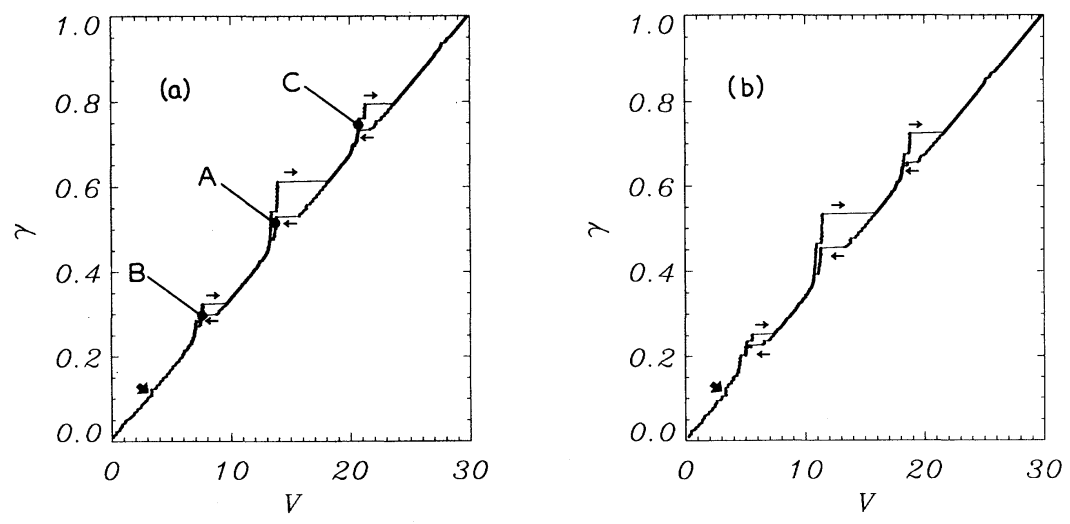

FIG. 1. Calculated $I-V$ characteristics of the long Josephson junction biased in the flux-flow state with the external oscillating field amplitude $a_{\mathrm{ext}}=2.5$ for $h=6.0$ (a) and $h=5.0(\mathrm{~b})$. 
fluxons $n_{\mathrm{fl}} \sim V_{f f} \approx 14$ is almost the same in the cases (a) $-(\mathrm{c})$.

The straight line $A_{1} A_{2}$ in Figs. 2(a)-(c) indicates the slope corresponding to the flux-flow mode trajectories [Fig. 2(a)] when fluxons flow homogeneously with almost maximum (Swihart) velocity. The comparison with $A_{1} A_{2}$ shows that in the LSS [Fig. 2(c)] fluxons move on average with the phase velocity lower than unity. All the trajectories contain periodically repeated almost vertical parts. The vertical part of a trajectory corresponds to the fluxon which does not move. In order to explain the physical mechanism related to the modulation of the trajectories we draw the line $B_{1} B_{2}$ which has the same slope as $A_{1} A_{2}$ but with the opposite spatial direction. One can notice that the standing-fluxon state propagates as a wave with Swihart velocity from $x=\ell$ towards $x=0$. The wave starts from $x=\ell$ at the moment when the external ac force $h_{\text {ext }}(t)$ has the maximum value. Because of the repulsive interaction between two unipolar magnetic fluxes (internal and external), the fluxon which was ready to leave the junction, being driven by the bias current, gets stopped close to the junction boundary. After a moment the next arrival also gets stopped by the first one, and later stops another one, etc. This sort of "shock-wave" propagates with Swihart velocity through the junction towards $x=0$. At the boundary $x=\ell$ the process repeats after the time interval $\Delta T_{\omega}=2 \pi / \omega_{\text {ext }} \approx 4.5$ when another wave is excited and starts propagating through the junction. Inside the junction, between the moments of time when the wave passes through a particular trajectory, the slope of the trajectory remains close to $A_{1} A_{2}$. When the wave crosses a trajectory, the trajectory moves upwards (the fluxon is stopped for a short time) and then this process is repeated with the next arriving wave. Because the spacing between fluxons changes periodically in response to the wave propagation, we denote these waves fluxon density waves.

In the HSS [Fig. 2(c)] the slope of trajectories is notice- ably lower than $A_{1} A_{2}$, which means that the phase velocity is larger than unity. In contrast to Fig. 2(b), now all the trajectories contain periodically repeated more horizontal parts. The line $C_{1} C_{2}$ drawn through these parts has the same slope as $B_{1} B_{2}$ in the previous figure. It starts at $x=\ell$ at the moment when the external ac force $h_{\text {ext }}(t)$ has the minimum value. At this moment, due to the boundary magnetic field the last fluxon gets pulled towards the boundary. Now instead of the compression wave (as in the case of the LSS) we find a sort of a rarification wave which starts propagating with Swihart velocity through the junction. As before, at the boundary $x=\ell$ the process is repeated with the time interval $\Delta T_{\omega}$. Inside the junction between passing density waves the slope of the trajectories remains close to that of $A_{1} A_{2}$. From Fig. 2(c) one can see that when the wave crosses any trajectory, the trajectory moves to the right and another trajectory follows the line $A_{1} A_{2}$.

The difference in dc voltages of the satellites from the FSS comes from the following. Every time the fluxon density wave passes through any trajectory in Fig. 2(c), the fluxon chain effectively shifts to the right by one fluxon period and another trajectory takes its place on the line $A_{1} A_{2}$. This gives one additional fluxon which contributes in the dc voltage of the junction once per period $\Delta T_{\omega}$. The process is started at $x=\ell$ when the external field pulls the fluxon from the junction. When the fluxon density wave arrives at $x=0$, it pulls an additional fluxon into the junction. At this moment the local density of trajectories is increasing. In order to make it more visible, the same time interval $\Delta T_{2}$ is marked in Fig. 2(a) and Fig. 2(c). One can see that during $\Delta T_{2}$ in the FFS two trajectories pass through the point $x=2$, whereas for the HSS there are three passing fluxon trajectories. Thus, due to the fluxon density wave propagation in a stationary regime per one period $\Delta T_{\omega}$ two additional fluxons contribute to dc voltage which becomes $V_{f f+1}=V_{f f}+2 V_{\omega}$. In a similar way, for the LSS every
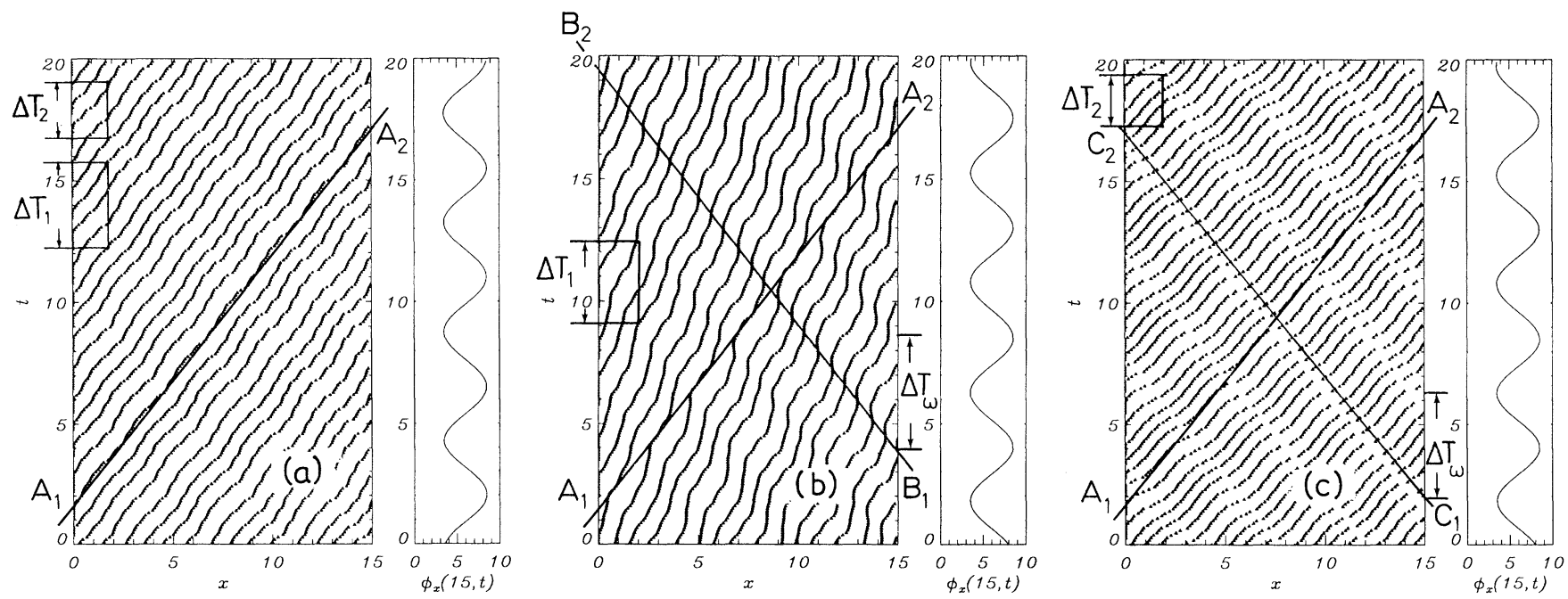

FIG. 2. Spatial-temporal fluxon trajectories through the long Josephson junctions in different bias points of the calculated $I$ - $V$ curves shown in Fig. 1. Each trajectory corresponds to $\varphi(x, t)=\varphi_{m}=2 \pi m+\pi$, where $m$ is an arbitrary integer. (a) point $A$, (b) point $B$, (c) point $C$. 
time the fluxon density wave passes through a trajectory [Fig. 2(c)] the flowing fluxon chain effectively shifts to the left by approximately one fluxon period. Once per period $\Delta T_{\omega}$ one fluxon gets stopped at $x=\ell$. The external force pushes the fluxon back into the junction. When the fluxon density wave arrives at $x=0$ it prevents another fluxon from coming into the junction. During $\Delta T_{1}$ in the FFS three trajectories cross the point $x=2$, whereas in the LSS there are only two crossing fluxon trajectories. In a stationary regime two fluxons do not contribute to flux-flow voltage during $\Delta T_{\omega}$, thus yielding $V_{f f-1}=V_{f f}-2 V_{\omega}$. From the energetical point of view, the HSS and LSS can be understood in the following way. For small bias current the fluxon energy is relatively small. In this case the external force can stop a fluxon close to the boundary $x=\ell$ (it cannot overcome the energy barrier $^{6}$ ) and the rarification wave appears, yielding the LSS. At the larger bias the FSS is still possible even in the presence of the ac force because its amplitude is not sufficient enough to stop fluxons at the boundary. For even larger bias current the ac force, oppositly, assists fluxons to overcome the barrier at $x=\ell$, which gives the HSS.

In Ref. 2 an explanation of HSS as due to microwaveassisted reflections of fluxons into antifluxons at the active junction boundary $(x=\ell)$ has been suggested. In this view, the line $C_{1} C_{2}$ in Fig. $2(\mathrm{c})$ can be treated as a trajectory of an antifluxon which propagates under the influence of the bias current in the opposite direction to the fluxon motion. Every time the antifluxon crosses the fluxon trajectory the phase difference is increasing by additional factor of $2 \pi$. This effectively shifts the fluxon trajectory to the right by one fluxon chain period. When the antifluxon arrives at $x=0$ it gets reflected back into the junction as a fluxon. Because $\Delta T_{\omega}$ and the time of fluxon flight across the junction are not necessarily com- mensurate, another reflection of this secondary fluxon from $x=\ell$ is not expected. Thus, for HSS we obtain two additional fluxon contributions the dc voltage per one pe$\operatorname{riod} \Delta T_{\omega}$, which again yields $V_{f f+1}=V_{f f}+2 V_{\omega}$. However, this rather simple fluxon-antifluxon model fails in explaining the LSS. Oppositely, the fluxon density wave approach presented above appears to be more general and works for the both HSS and LSS regimes.

Finally, one more thing requires to be commented on. In the HSS regime the fluxon trajectories $\varphi_{m}$ move with the average velocity $v_{m}>1$ [Fig. 2(c)]. Conventionally, fluxons are assumed to be stable only if their velocity $v$ is below unity. In general, the nonperturbed sineGordon equation has two types of single-soliton solutions, (i) $\varphi=4 \tan ^{-1}\left\{\exp \left[(x-v t) / \sqrt{1-v^{2}}\right]\right\}$ for $v<1$, and (ii) $\varphi=\pi-4 \tan ^{-1}\left\{\exp \left[(x-v t) / \sqrt{v^{2}-1}\right]\right\}$ for $v>1$. In the simplest cases the second solution (ii) is known to be unstable. However, in the flux-flow regime which we consider here the phase difference shift between the junction boundaries is $\Delta \varphi=\varphi(L)-\varphi(0) \approx h \ell \neq 0$. With the increase of the bias current $\gamma \rightarrow 1$ the conventional soliton mode of type (i) with $v<1$ becomes unstable and the system jumps into another state of the spatially dependent phase rotation with the type (ii) solution for $v>1$. The solution (ii) is stabilized by the topological restriction $\Delta \varphi \neq 0$ placed on the system. Perhaps, one might be able to find a theoretical approach which treats the fluxon density wave in the HSS regime similarly to that in the LSS using the symmetry between (i) and (ii) type of solutions.

We are grateful to S. Benz, M. Cirillo, Yu. S. Kivshar, B. H. Larsen, B. A. Malomed, J. Mygind, S. Pagano, and M. R. Samuelsen for useful discussions. This work was partially supported by NATO Grant No. LG-920672.
*Permanent address: Institute of Solid State Physics, Russian Academy of Sciences, Chernogolovka, Moscow district, 142432, Russia.

${ }^{1}$ N. F. Pedersen, IEEE Trans. Magn. MAG-27, 3328 (1991).

${ }^{2}$ A. V. Ustinov, J. Mygind, N. F. Pedersen, and V. A. Oboznov, Phys. Rev. B 46, 578 (1992).

${ }^{3} \mathrm{H}$. Olsson and T. Claeson, in Proceedings of the Low Tem- perature Physics Conference, edited by U. Eckern et al. (Elsevier, New York, 1984), p. 213.

${ }^{4}$ T. Nagatsuma, K. Enpuku, K. Yoshida, and F. Irie, J. Appl. Phys. 56, 3284 (1984).

${ }^{5}$ S. Shapiro, Phys. Rev. Lett. 11, 80 (1963).

${ }^{6}$ B. A. Malomed (private communication). 